\title{
A GLOBAL EXISTENCE THEOREM FOR SMOLUCHOWSKI'S COAGULATION EQUATIONS
}

\author{
WARREN H. WHITE
}

ABSTRACT. A countable system of nonlinear ordinary differential equations is shown to admit solutions valid over all positive time.

Consider the following countable system of equations on $[0, \infty), k=1,2, \ldots$ [1], [2], [3]:

$$
\begin{gathered}
\frac{d x_{k}}{d t}-c_{k}=\frac{1}{2} \sum_{i+j=k} b_{i j} x_{i} x_{j}-x_{k} \sum_{j} b_{k j} x_{j}, \\
x_{k}(0)=a_{k}>0,
\end{gathered}
$$

where the $b_{i j}$ and $c_{k}$ are continuous functions of $t$ such that $b_{i j}=b_{j i}>0$ and $c_{k}>0$. The $b_{i j}$ arising in applications typically increase without bound as $i$ and $j$ increase, so that (1) is beyond the reach of standard results on differential equations in Banach spaces. The case $b_{i j}<i j$ has been studied by McLeod [4], [5], who established the existence and uniqueness of a nonnegative $\left(x_{k}>0\right)$ initial solution which does not, in general, extend to $t>1$. The $b_{i j}$ arising in most applications satisfy the more restrictive condition $b_{i j}<i+j$, for which case the present note demonstrates the existence of global, well-behaved solutions.

TheOREM 1. Let $b_{i j} \leqslant i^{\alpha}+j^{\alpha}$ for some $\alpha \in[0,1]$. Let $\Sigma_{k} k^{p} a_{k}$ be finite, and $\Sigma_{k} k^{p} c_{k}$ bounded on bounded intervals, for some integer $p>\alpha$. Then (1) admits at least one nonnegative solution on $[0, \infty)$. For any nonnegative solution $x, \Sigma_{k} k^{p} x_{k}$ is bounded on bounded intervals.

The proof of Theorem 1 begins with a technical definition abstracting conditions necessary to carry through McLeod's local arguments [4] on a global basis. It will be convenient to denote sequences and kernels by the unsubscripted symbols used for their elements (e.g., $\left(a_{k}\right)=a,\left(b_{i j}\right)=b$ ), and to write $b^{\prime}<b$ to indicate that $b_{i j}^{\prime}<b_{i j}$ for all $i$ and $j$. With these conventions, let $S_{a}(b, c)$ be the (possibly empty) set of nonnegative solutions to $(1)$ on $[0, \infty)$.

Definition. Problem (1) has the bounded $\beta$-moment property for some $\beta \in$ $[0, \infty)$ if there exists a continuous function $m_{\beta}:[0, \infty) \rightarrow[0, \infty)$ such that $\Sigma_{k} k^{\beta} x_{k}$ $<m_{\beta}$ for all $x \in \cup_{b^{\prime}<b} S_{a}\left(b^{\prime}, c\right)$.

Lemma 2. Let $b_{i j}<B i^{\alpha} j^{\alpha}$ for some $\alpha \in[0, \infty)$. If (1) has the bounded $\beta$-moment property for some $\beta>\alpha$, then it admits at least one nonnegative solution on $[0, \infty)$.

Received by the editors October 29, 1979.

1980 Mathematics Subject Classification. Primary 34A10; Secondary 45J05, 70F35. 
Proof. The proof of this lemma closely follows the approach taken by McLeod in the proof of his Theorem 2 [4].

For each positive integer $N$, define a truncated kernel $b^{(N)}$ as follows.

$$
b_{i j}^{(N)}= \begin{cases}b_{i j} & \text { for } i+j<N \\ 0 & \text { for } i+j>N\end{cases}
$$

Straightforward finite-dimensional arguments show the solution set $S_{a}\left(b^{(N)}, c\right)$ to consist of a single element, $x^{(N)}$. Assuming (1) to have the bounded $\beta$-moment property, there exists a continuous function $m_{\beta}:[0, \infty) \rightarrow[0, \infty)$ such that $m_{\beta}>$ $\Sigma_{k} k^{\beta} x_{k}^{(N)}$ for each $N$. Since the $x^{(N)}$ are nonnegative, $x_{k}^{(N)}$ and $d x_{k}^{(N)} / d t$ are then bounded on bounded intervals, uniformly for all $N$ :

$$
\begin{aligned}
x_{k}^{(N)} & <\sum_{j} j^{\beta} x_{j}^{(N)}<m_{\beta}, \\
\left|\frac{d}{d t} x_{k}^{(N)}\right| & =\left|\frac{1}{2} \sum_{i+j=k} b_{i j}^{(N)} x_{i}^{(N)} x_{j}^{(N)}-x_{k}^{(N)} \sum_{j} b_{k j}^{(N)} x_{j}^{(N)}+c_{k}\right| \\
& <\frac{B}{2} \sum_{i} \sum_{j} i^{\beta} j^{\beta} x_{i}^{(N)} x_{j}^{(N)}+B k^{\beta} x_{k}^{(N)} \sum_{j} j^{\beta} x_{j}^{(N)}+c_{k}<2 B m_{\beta}^{2}+c_{k} .
\end{aligned}
$$

Ascoli's lemma can therefore be applied, repeatedly, to obtain an increasing sequence $J$ of integers such that $\left\{x_{k}^{(N)}: N \in J\right\}$ is uniformly convergent on bounded intervals for each $k$. In what follows, $N$ will be restricted to values in $J$.

Let $x_{k}=\lim _{N \rightarrow \infty} x_{k}^{(N)}$ for each $k$. To verify equation (1a) in the limit, it suffices to show that $\sum_{j} b_{k j}^{(N)} x_{j}^{(N)}$ converges to $\sum_{j} b_{k j} x_{j}$ uniformly on bounded intervals. For each $k$,

$$
\begin{aligned}
\mid \sum_{j} b_{k j} x_{j}- & \sum_{j} b_{k j}^{(N)} x_{j}^{(N)} \mid \\
& <\sum_{j<N_{1}}\left|b_{k j} x_{j}-b_{k j}^{(N)} x_{j}^{(N)}\right|+\sum_{N_{1}<j}\left(b_{k j} x_{j}+b_{k j}^{(N)} x_{j}^{(N)}\right) .
\end{aligned}
$$

The second term on the right-hand side can be made arbitrarily small by choosing $N_{1}$ large:

$$
\begin{aligned}
\sum_{N_{1}<j}\left(b_{k j} x_{j}+b_{k j}^{(N)} x_{j}^{(N)}\right) & <B k^{\alpha} \sum_{N_{1}<j} j^{\alpha}\left(x_{j}+x_{j}^{(N)}\right) \\
& <B k^{\alpha} \sum_{N_{1}<j} N_{1}^{\alpha-\beta_{j} \beta}\left(x_{j}+x_{j}^{(N)}\right)<2 B k^{\alpha} m_{\beta} N_{1}^{\alpha-\beta}
\end{aligned}
$$

The first term on the right-hand side of (2) can be made arbitrarily small-for fixed $N_{1}$-by choosing $N$ large.

Proposition 3. Let $x$ be a nonnegative solution to (1). Then

$$
\frac{d}{d t} \sum_{k<N} k^{p} x_{k}-\sum_{k<N} k^{p} c_{k} \leqslant \frac{1}{2} \sum_{1<q<p-1}\left(\begin{array}{l}
p \\
q
\end{array}\right) \sum_{i<N} \sum_{j<N} i^{q} j^{p-q} b_{i j} x_{i} x_{j}
$$

for all positive integers $p$ and $N$. 
Proof. From equation (1a),

$$
\begin{aligned}
\frac{d}{d t} \sum_{k<N} k^{p} x_{k}-\sum_{k<N} k^{p} c_{k}= & \sum_{k<N} k^{p}\left(\frac{d x_{k}}{d t}-c_{k}\right) \\
= & \frac{1}{2} \sum_{k<N} k^{p} \sum_{i+j=k} b_{i j} x_{i} x_{j}-\sum_{k<N} k^{p} x_{k} \sum_{j} b_{k j} x_{j} \\
= & \frac{1}{2} \sum_{k<N} \sum_{i+j=k}(i+j)^{p} b_{i j} x_{i} x_{j}-\sum_{k<N} \sum_{j} k^{p} b_{k j} x_{k} x_{j} \\
= & \frac{1}{2} \sum_{k<N} \sum_{i+j=k} \sum_{1<q<p-1}\left(\begin{array}{l}
p \\
q
\end{array}\right) i^{q} p^{p-q} b_{i j} x_{i} x_{j} \\
& +\sum_{k<N} \sum_{i+j=k} i^{p} b_{i j} x_{i} x_{j}-\sum_{k<N} \sum_{j} k^{p} b_{k j} x_{k} x_{j} \\
= & \frac{1}{2} \sum_{k<N} \sum_{i+j=k} \sum_{1<q<p-1}\left(\begin{array}{l}
p \\
q
\end{array}\right)^{i q j p-q} b_{i j} x_{i} x_{j} \\
& -\sum_{k<N} \sum_{N-k<j} k^{p} b_{k j} x_{k} x_{j} .
\end{aligned}
$$

The desired inequality follows from the hypothesis that $x$ is nonnegative:

$$
\begin{aligned}
\frac{d}{d t} \sum_{k<N} k^{p} x_{k}-\sum_{k<N} k^{p} c_{k} & <\frac{1}{2} \sum_{k<N} \sum_{i+j=k} \sum_{1<q<p-1}\left(\begin{array}{c}
p \\
q
\end{array}\right) i^{q} q^{p-q} b_{i j} x_{i} x_{j} \\
& <\frac{1}{2} \sum_{1<q<p-1}\left(\begin{array}{c}
p \\
q
\end{array}\right) \sum_{i<N} \sum_{j<N} i^{q}{ }^{p-q} b_{i j} x_{i} x_{j} .
\end{aligned}
$$

LEMMA 4. Let $b_{i j}<i+j$. Let $\Sigma_{k} k^{p} a_{k}$ be finite, and $\Sigma_{k} k^{p} c_{k}$ bounded on bounded intervals, for some positive integer $p$. Then (1) has the bounded p-moment property.

Proof. Define

$$
\begin{aligned}
& m_{1}(t)=\int_{0}^{t} \sum_{k} k c_{k}(\tau) d \tau+\sum_{k} k a_{k}, \quad w(t)=\int_{0}^{t} m_{1}(\tau) d \tau, \\
& m_{2}(t)=e^{2 w(t)}\left\{\int_{0}^{t} e^{-2 w(\tau)} \sum_{k} k^{2} c_{k}(\tau) d \tau+\sum_{k} k^{2} a_{k}\right\} \\
& m_{p}(t)=e^{p w(t)}\left\{\int _ { 0 } ^ { t } e ^ { - p w ( t ) } \left(\sum_{1<q<p-2}\left(\begin{array}{l}
p \\
q
\end{array}\right) m_{q+1}(\tau) m_{p-q}(\tau)\right.\right. \\
& \left.\left.+\sum_{k} k^{p} c_{k}(\tau)\right) d \tau+\sum_{k} k^{p} a_{k}\right\}, \quad p>2 .
\end{aligned}
$$

The $m_{p}$ are continuous, because the integrands involved in their definition are bounded. Let $x \in \cup_{b^{\prime}<b} S_{a}\left(b^{\prime}, c\right)$; it will be shown, by induction on $p$, that $\sum_{k} k^{P} x_{k}<m_{p}$.

For $p=1$, the right-hand side of (3) is zero, so that

$$
\sum_{k<N} k x_{k}<\int_{0}^{t} \sum_{k<N} k c_{k}(\tau) d \tau+\sum_{k<N} k a_{k}<m_{1}(t)
$$


for all $N$. Letting $N$ increase yields the desired result. Suppose now that $\Sigma_{k} k^{q} x_{k}<$ $m_{q}$ for all $q<p, p \geqslant 2$. Substitution of the bound $b_{i j}<i+j$ into (3) gives

$$
\begin{aligned}
\frac{d}{d t} \sum_{k<N} k^{p} x_{k} & \leqslant \sum_{1<q<p-1}\left(\begin{array}{l}
p \\
q
\end{array}\right) \sum_{i<N} i^{q+1} x_{i} \sum_{j<N} j^{p-q} x_{j}+\sum_{k<N} k^{p} c_{k} \\
& <p m_{1} \sum_{k<N} k^{p} x_{k}+\sum_{1<q<p-2}\left(\begin{array}{c}
p \\
q
\end{array}\right) m_{q+1} m_{p-q}+\sum_{k} k^{p} c_{k} .
\end{aligned}
$$

Integration of this inequality gives $\sum_{k<N} k^{p} x_{k}(t)<m_{p}(t)$ for all $N$. Letting $N$ increase again yields the desired result.

Proof OF Theorem 1. Lemma 4 establishes that (1) has the bounded $p$-moment property under the hypotheses of Theorem 1 (since $i^{\alpha}+j^{\alpha}<i+j$ ). By Lemma 2 (since $i^{\alpha}+j^{\alpha} \leqslant 2 i^{\alpha} j^{\alpha}$ ), (1) therefore admits at least one nonnegative solution on $[0, \infty)$. By definition of the bounded $p$-moment property, the $p$-moment of any nonnegative solution is bounded on bounded intervals.

\section{REFERENCES}

1. M. v. Smoluchowski, Versuch einer mathematischen Theorie der koagulationskinetik kolloider Losungen, Z. Phys. Chem. 92 (1918), 129-168.

2. R. L. Drake, A general mathematical survey of the coagulation equation, Topics in Current Aerosol Research 3 (Part 2), G. M. Hidy and J. R. Brock (eds.), Pergamon, Oxford, 1972, pp. 201-376.

3. H. R. Pruppacher and J. D. Klett, Microphysics of clouds and precipitation, Reidel, Dordrecht, 1978.

4. J. B. McLeod, On an infinite set of nonlinear differential equations, Quart. J. Math. Oxford Ser. (2) 13 (1962), 119-128.

5. (1962), 193-205.

CAPITA, Box 1185, Washington University, St. Louis, Missouri 63130 